\title{
Experimental Evaluation of Ability of Relap5 and Drako@ to Calculate Water Hammer with Phase Changes
}

AUTHORS:

Jerzy Marcinkiewicz (corresponding author), Inspecta Nuclear AB, Stockholm, Sweden Adam Adamkowski, Mariusz Lewandowski, The Szewalski Institute o Fluid-Flow Machinery of the Polish Academy of Sciences (IMP PAN), Gdansk, Poland.

\section{Summary:}

A proper calculations of loadings caused by water hammer (hydraulic transients) occurring in pipeline systems in nuclear power plants are very important issue according to systems viability, safety and reliability. Calculating the water hammer loadings on pipe sections according to the classical one-dimensional (1D) theory of liquid transient flow in a pipeline, and then transferring the results to strength analyses of pipeline structure (walls), is nowadays the most common calculation procedure used in Sweden. This procedure assumes that the respond of the pipeline structure to the pressure surges is quasi-steady - dynamic interaction between the fluid and the pipeline construction is set aside.

For calculating the hydraulic loadings in the 1D domain so-called network programs are used - Relap5 and Drako are the representatives of this kind of programs which are the most commonly used in Sweden nowadays. As a third party accredited inspection body INSPECTA NUCLEAR AB reviews all calculations of water hammer loadings. A question of vital importance for the reviewer is the knowledge about the ability of the programs to calculate the loadings under different conditions. While Relap5 and Drako calculations of the loadings without phase changes have been investigated quite well, the ability of the programs to calculate the loadings when phase changes occur cannot be considered to be sufficient.

The presented work shall be seen as an attempt to illustrate the ability of Relap5 and Drako programs to calculate the water hammer with phase changes. Some parametric analyses have been performed in order to investigate the influence of digitizing of time and space. All differences between the results obtained with Relap5 and Drako are discussed.

The calculations are compared with experimental results. The experiments have been conducted at a test rig designed and constructed at the Szewalski Institute of Fluid-Flow Machinery of the Polish Academy of Sciences (IMP PAN) in Gdansk, Poland.

The comparisons of calculated and measured pressure surges show significant differences between them. The discrepancies are discussed and conclusions are presented. 
ICONE15-10326

\section{EXPERIMENTAL EVALUATION OF ABILITY OF RELAP5 AND DRAKO® TO CALCULATE WATER HAMMER WITH PHASE CHANGES}

\author{
Jerzy Marcinkiewicz \\ Inspecta Nuclear $A B$ \\ SE-104 25 Stockholm, Sweden \\ Phone: +46-8-50113065 \\ Fax: +46-8-50113001 \\ jerzy.marcinkiewicz@inspecta.com
}

\author{
Adam Adamkowski \\ Mariusz Lewandowski \\ The Szewalski Institute of Fluid Flow \\ Machinery, Gdansk, Poland \\ aadam@imp.gda.pl \\ mlew@imp.gda.pl
}

Keywords: water hammer, phase changes, water column separation, dynamic loadings, Relap5, Drako.

\section{ABSTRACT}

Mechanical loadings on pipe systems caused by water hammer with phase changes make calculation of final forces difficult in nuclear power plants. The common procedure in Sweden is to calculate the water hammer loadings, according to the classical one-dimensional theory of liquid transient flow in a pipeline, and then transfer the results to strength analyses of pipeline structure. This procedure assumes that there is quasisteady response of the pipeline structure to pressure surges - no dynamic interaction between the fluid and the pipeline construction. The hydraulic loadings are calculated with 1-D so-called "network" programs. Commonly used in Sweden are Relap5 (Mod3.2.2 and higher) and Drako. As a third party accredited inspection body INSPECTA NUCLEAR AB reviews calculations of water hammer loadings. An important question for the reviewer (and also for the users) is knowledge about their ability to calculate the dynamic loadings. While the ability of Relap5 and DRAKO to calculate water hammer without phase changes is
\end{abstract}

relatively well investigated the skills of the programs when phase changes are present need some more attention. The presented work shall be seen as an attempt to illustrate ability of Relap5, and Drako programs to calculate the water hammer loadings with phase changes. A special attention was paid to using of Relap5 for calculation of water hammer pressure surges (including some aspects of influence of discretisation of space on the calculation results). The calculations are compared with experimental results. The experiments have been conducted at a test rig designed and constructed at the Szewalski Institute of Fluid-Flow Machinery of the Polish Academy of Sciences (IMP PAN) in Gdansk, Poland. The comparison of calculated and measured pressures shows some differences, only the first pressure peak, occurring before evaporation is calculated quite exactly. All next coming pressure peaks differ slightly from the measured with respect to amplitude and frequency. 


\section{INTRODUCTION}

According to rules stated by Swedish regulatory body (SKI) the calculated loadings must be reviewed and accepted by a notified third party inspection body. Inspection Nuclear $\mathrm{AB}$ performs such work. The purpose of the review is to confirm that the loadings are not underestimated. Moreover, a number of key technical requirements which influence the review process are stated in the SKIFS-series [1] like the following:

- well-tried, verified and recognized methods/codes with good safety margins shall be used,

- well-founded input data and prudent conservative assumptions shall be used, and also the latest knowledge/experience shall be considered when calculating the loadings.

Several computer programs are used in Sweden for the calculation of dynamic loadings on pipe systems caused by fluid transients. These kinds of calculations were at the beginning dominated by large companies: ASEA/ABB Atom, Westighouse Electric, General Electric, Siemens and Areva. Today, because of availability of software (Relap5 and Drako) small consultant companies can also perform such analyses. When the calculation can be performed with several computer programs an important question for the reviewer (and also for the users) is knowledge about their ability to calculate the dynamic loadings and eventual differences. The ability of the basic commercial programs (Relap5 and Drako) to calculate water hammer in cases without phase changes (without liquid column separations) was presented in [6]. Furthermore it is very important to perform study of ability of the programs to calculate pressure surges in a pipeline when phase changes occur and compare with experimental data. Such cases belong to the most difficult to calculate design loadings in nuclear power plants. Two versions of Relap5 (Mod3.2.2g and Mod3.3patch02) and version 3.22 of Drako were considered in this paper.

\section{EXPERIMENTS}

Experiments were conducted at a test stand specially prepared in order to investigate the water hammer phenomenon - Fig.1. The stand was constructed at the laboratory of IMP PAN in Gdansk (Poland). Its main component is the $L=98.11 \mathrm{~m}$ long copper pipe with internal diameter of $D=0.016 \mathrm{~m}$ and wall thickness of $e=0.001 \mathrm{~m}$. The pipe is spirally coiled on a steel cylinder with diameter of about $1.7 \mathrm{~m}$ and is rigidly mounted to the steel cylinder coating in order to minimize its vibrations. The inclination angle of the pipe $(\beta)$ is not larger than $0.5^{\circ}$. A quick-closing ball valve is installed at one end of the pipe. The special spring drive of this valve enables an almost stepwise complete flow cut-off. This has been confirmed by the records of valve closure with duration not exceeding $0.003 \mathrm{~s}$, which is about $1 \%$ of the period of pressure wave propagation along the pipe $(4 L / a)$

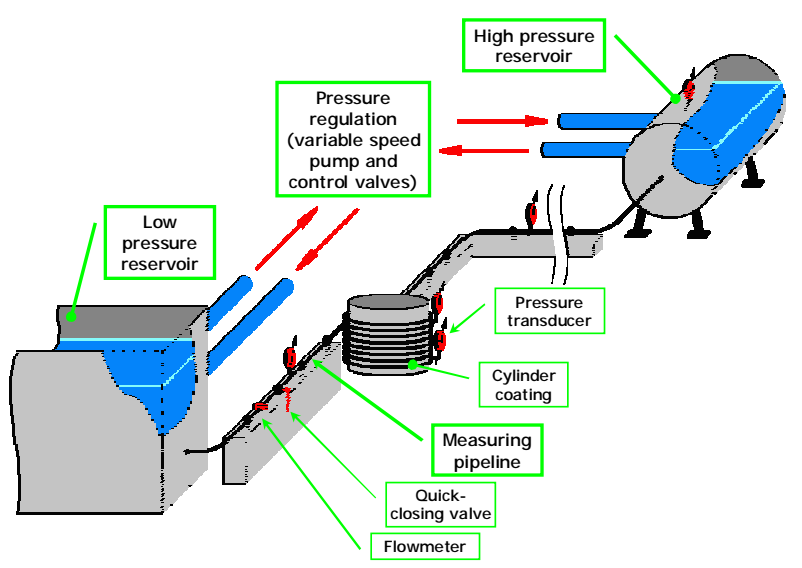

Figure 1. Layout of the test rig

The test rig was equipped with appropriate measuring instrumentation. Four absolute pressure semi-conductor transducers were mounted at equidistant (every $L / 4$ ) sections along the pipeline. The measuring range of these transducers was (0-4) MPa with transmitted frequency band: $(0-2) \mathrm{kHz}$ and precision class: $0.2 \%$. The transmitted frequency band of the transducers, $0-2 \mathrm{kHz}$, indicates that the rise time of output signal for up to $4 \mathrm{MPa}$ stepwise change of pressure (measuring range) is shorter then $0.0005 \mathrm{~s}$ $(1 / 2000 \mathrm{~Hz})$. This time is shorter than about $0.17 \%$ of the pressure wave period $(4 L / a=\sim 0.3 \mathrm{~s})$ or, in other words, transmitted frequency band for each transducer is at least 600 times wider then pressure wave frequency equal about $3.3 \mathrm{~Hz} \quad(a /(4 L))$. A turbine flowmeter with the range of $1.5 \mathrm{~m}^{3} / \mathrm{h}$ $\left(\sim 4.2 \cdot 10^{-4} \mathrm{~m}^{3} / \mathrm{s}\right)$ and precision class of $1 \%$ is used for indirect measurement of flow velocity in the pipeline.

Before starting the experiments, the hydraulic system was filled with water and left for a few days to remove undissolved air from the liquid in as much as possible. The intention was to minimize the influence of undissolved air bubbles on the considered phenomenon courses ${ }^{1}$.

The experimental test consisted in several runs of pipe unsteady flow after sudden valve closure. Water hammer without or with accompanying water column separation was generated depending on the initial flow conditions - velocity $V_{\mathrm{o}}\left(\mathrm{Re}_{\mathrm{o}}\right)$ and level $H_{\mathrm{o}}$ in the highpressure reservoir. Because of the limited value of $H_{\mathrm{o}}$ the test stand enables to generate water hammer without column separation phenomenon at initial conditions of Reynolds numbers up to $\mathbf{R e}_{\mathbf{0}} \cong 16000$. (The kinematic viscosity coefficient was specified $\left(v=9.432 \cdot 10^{-7} \mathrm{~m}^{2} / \mathrm{s}\right)$ for the water temperature kept about $22.6^{\circ} \mathrm{C}$ during test.)

\footnotetext{
${ }^{1}$ Naturally such an operation did not assure removing amounts of air released under low pressure occurred during column separation phenomenon (gas cavity).
} 
Every test run was started by adjustment of required stable initial conditions (initial velocity $V_{\mathrm{o}}$ and steady head water level $H_{\mathrm{o}}$ ). Then, after some period needed for stabilization of the flow conditions (not less then $180 \mathrm{~s}$ ), the flow rate measurement was conducted. Recording of the pressure changes and the valve closure rate was started a few seconds before quick cutting-off the flow (water hammer phenomenon). Flow cut-off was executed by releasing the spring in the driving mechanism of the quickclosing valve. After the recording was ended the test rig was prepared to execute next experimental run.

Figure 2 presents selected samples of pressure variations measured for two different cases (runs): a) the first one - for water hammer without column separation, b) the second one - for water hammer with column separation. Pressure changes in these two cases have different nature and also different range.

The case presented on figure 2a) shows typical situation for water-hammer phenomenon without water column separation. The reduced pressure changes have constant frequency and are approximately symmetric in relation to the pressure level in the upper reservoir. Minimal pressure is higher than vapour pressure for water at the temperature at which the tests were conducted.

On the contrary, figure $2 \mathrm{~b}$ ) shows pressure courses characteristic for water-hammer with accompanying water column separation. After the first pressure increment (pressure peak), caused by stepwise cut-off of the flow, pressure measured at the valve falls to the critical value close to vapour pressure and stays at this level for longer time than it follows from the pressure wave period.

During this time vapour generation occurs and when it decays instant pressure increment of high value appears. Such pressure pulse repeat cyclically with decreasing amplitudes and with increasing frequency. These pressure changes are accompanied by intensive acoustic effects - resembling knocking noise. After some time the pressure peaks are transformed into oscillatory changes typical for waterhammer without water column separation (pressure higher than vapour pressure and constant frequency).

For the further consideration, pressure wave speed $a$ was calculated from the data recorded ${ }^{2}$ for the cases of water hammer without column separation ${ }^{3}$. For each considered run the value of wave period, $4 L / a$, was determined basing on 20 pressure oscillations. The mean value of pressure wave speed, calculated from taken into account tested runs was equal $a=1297 \mathrm{~m} / \mathrm{s}$.

\footnotetext{
${ }^{2}$ The main reason for calculating $a$ from the experimental data was to avoid its influence on comparisons between calculated and measured pressure courses.

${ }^{3}$ Releasing air from water under low pressure, especially during liquid column separation, causes significant decrease of the pressure wave speed $a$.
}
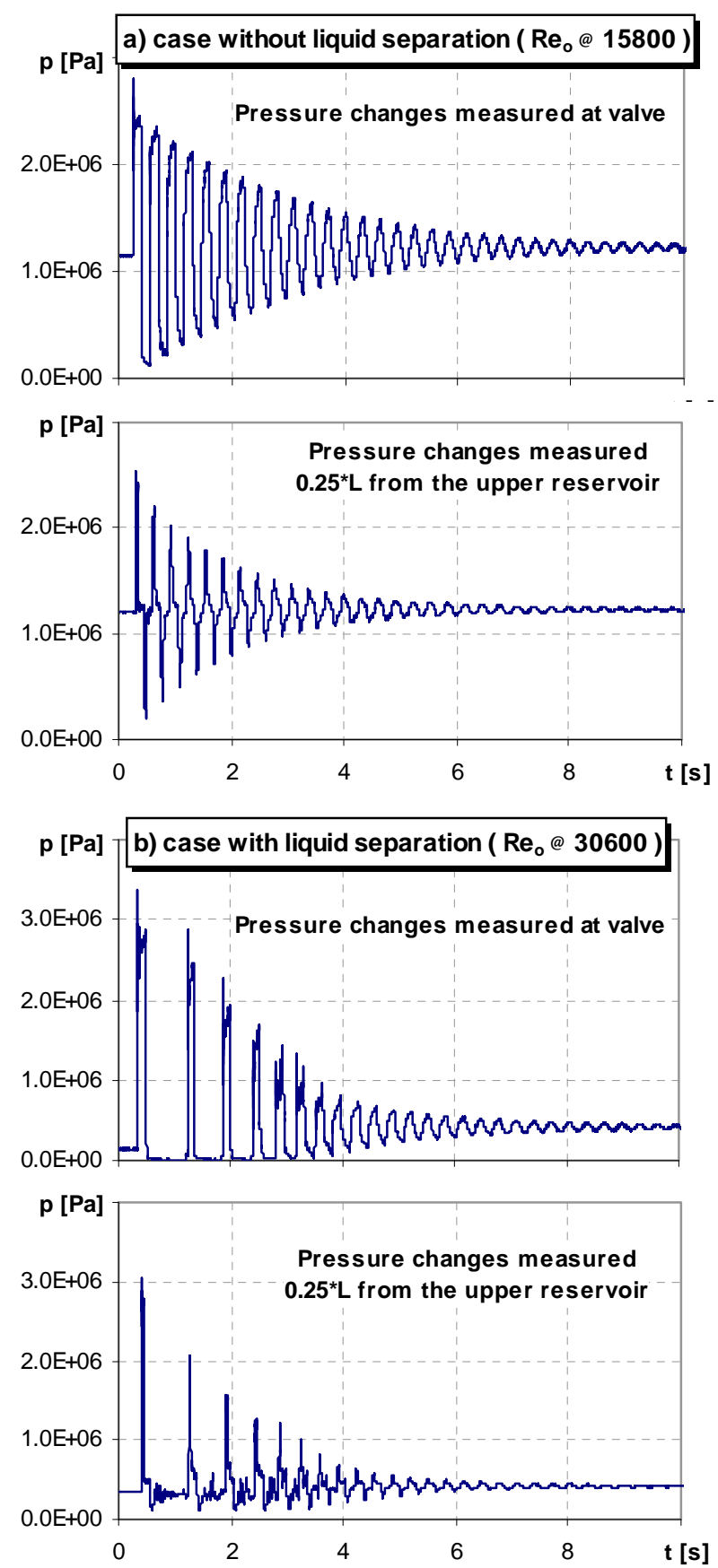

Figure 2. Pressure changes measured at the valve and at pipe cross-section located $0.25 * L$ from the upper reservoir: a) case without liquid separation, b) case with liquid separation

It should be emphasized that this value of $a$ (the mean pressure wave speed determined basing on the experimental data) coincides very well with the value calculated from the following formula $[8]^{4}$ : It was assumed that the pipeline at the test rig is anchored against
longitudinal movement 


$$
a=\frac{\left(K_{w} / \rho\right)^{0.5}}{\left[1+\left(1-v^{2}\right) \frac{K_{w}}{E} \frac{D}{e}\right]^{0.5}}=1298.4 \mathrm{~m} / \mathrm{s}
$$

where:

$$
\begin{aligned}
& \text { for water at } \sim 20^{\circ} \mathrm{C} \text { : } \\
& \text { bulk modulus } \quad K_{w}=2.1 \cdot 10^{9} \mathrm{~Pa} \\
& \text { density } \quad \rho=1000 \mathrm{~kg} / \mathrm{m}^{3} \text {, } \\
& \text { for copper pipeline coating: } \\
& \text { Poisson coefficient } \quad v=0.35 \text {, } \\
& \text { Young modulus: } \quad E=120 \cdot 10^{9} \mathrm{~Pa} \text {, } \\
& \text { internal pipe diameter: } D=0.016 \mathrm{~m} \text {, } \\
& \text { pipe wall thickness: } \quad e=0.001 \mathrm{~m} \text {. }
\end{aligned}
$$

\section{CALCULATIONS WITH RELAP5, DRAKO AND COMPARISON WITH EXPERIMENTAL DATA}

Relap5 code [3] has been developed for bestestimate transient simulations in light water reactors coolant systems during postulated accidents but is also used for thermal hydraulic analyses in nuclear power plants for processes which have time scale of magnitude about 1 second. Code uses include analyses required to support rulemaking, licensing audit calculations, evaluation of accident mitigation strategies, evaluation of operator guidelines and experiment planning analysis. The Relap5Mod3 code is based on a non-homogeneous and non-equilibrium model for two phase system that is solved by a fast, partially implicit numerical scheme. Two versions of Relap5 code were used in the presented analyses: Mod3.2.2g (released 1999) and Mod3.3Patch02 (released 2004).

A special attention regarding choice of time step is recommended by Relap5 developing group when very rapid processes like steam and water hammer are to be calculated (see chapter 2.1.2.2 in [4]). Correct use of Relap5 for analysing very rapid processes need application of proper cell size to resolve the wave front and time step small enough to meet the Courant limit and to minimize numerical diffusion $[5,6]$. It should be observed that using too small time step can bring numerical oscillations and in some cases lead to failure of the simulation [5].

It is also important to point out that Relap5 is not the most appropriate or user friendly code for solving water hammer problems but it is well documented, easy to access and able to produce reliable results, and therefore is commonly used for such purposes in Sweden.

Drako code [7, www.kae-gmbh.de ] has been specially developed for numerical prediction of unsteady flow processes in complex pipe systems and is commonly used for calculation of water and steam hammer loadings in nuclear power plants.

Three procedures can be used by Drako to solve the differential equation describing the propagation of pressure waves [7]:

- the method of characteristics,

- the differential procedure based on McCormack - this procedure is a finite differential method of the $2^{\text {nd }}$ order (noncentered difference scheme for nonlinear hyperbolic equations),

- Taylor $1^{\text {st }}$ order gives quite large numerical diffusion and is not recommended for analyses with steep gradients.

In addition to three conservation equations for mass, momentum and energy Drako uses a material law to describe the thermodynamic state variables in form of density as a function of pressure and entropy and enthalpy as a function of pressure and entropy. The material model for water and steam is based on the "IAPWS Industrial Formulation 1997 for the Thermodynamic Properties of Water and Steam (IAPWS-IF97)". Drako is based on homogeneous equilibrium model for two phase systems. Any detailed information about modelling of water column separation and rejoining is not available in program documentation.

\subsection{Conditions and assumptions valid for Relap5 and Drako models}

The calculation models are similar to the test stand. The case corresponding to $\mathrm{Re}_{0}=30661$ is analysed, i.e. initial velocity is $1.82 \mathrm{~m} / \mathrm{s}$ and water temperature $22.6^{\circ} \mathrm{C}$. The small increase in wall friction caused by curvature of the pipeline is generally not considered. In order to make possible direct comparisons of calculated pressures, the following small modifications of input data for Relap5 and Drako have been made:

- Relap 5. Because modifying of pressure wave speed is not possible in the program the pipe length and initial velocities has been changed (multiplied respectively divided by factor $1500 / 1297$, where 1500 $\mathrm{m} / \mathrm{s}$ is the sonic speed in the water at $23^{\circ} \mathrm{C}$ and 1297 $\mathrm{m} / \mathrm{s}$ is the pressure wave speed measured in the test rig). The change of Reynolds number caused by this modification is judged be negligible for the results.

- Drako calculates the wave speed automatically considering the elasticity of pipe wall. The measured wave speed could not be exactly reached in the calculations because of simplified formula used by Drako (not considering Poisson's coefficient). To reach the wave speed of $1297 \mathrm{~m} / \mathrm{s}$ the Young modulus of pipe wall material has been slightly changed. To solve equations the McCormack algorithm is used in spite of the fact that Drako manual recommends using the method of characteristics for solving water hammer where large changes in density occur. The reasons for choosing McCormack was to investigate if also this solver can satisfactory manage such problems.

The additional difficulty with Drako is that the steam tables are covering temperatures from $50^{\circ} \mathrm{C}$ and 
higher. Own data covering region under $50^{\circ} \mathrm{C}$ were not introduced. Instead temperature of $50^{\circ} \mathrm{C}$ is used and static pressure in the pipeline has been increased with value corresponding to difference in saturation pressures between $23^{\circ} \mathrm{C}$ and $50^{\circ} \mathrm{C}$ (i.e. stagnation pressures in the reservoirs has been increased with 0.097 bar). Moreover - the change of temperature will slightly increase (by $2.5 \%$ ) the speed of sound in the water. This deviation has not been taken into account.

Following scenario is valid for the calculations:

- between 0 and 5.5 seconds stationary conditions are calculated,

- the valve closes at $\mathrm{t}=5.5 \mathrm{~s}$ and closing time is $0.003 \mathrm{~s}$.

- calculations continue to time point $t=8.5 \mathrm{~s}$ with exception to Relap5 calculations with smallest cell lengths $(0.0725 \mathrm{~m})$ which are extremely time consuming and therefore the calculations are stopped earlier (between 7.2 and 8)

\subsection{Pressures calculated with Relap5 and Drako}

A special attention has been paid to Relap5 calculations because of sensitivity of the program to numerical damping. According to recommendations in manual and $[5,6]$ Courant number based on wave and bulk velocity, equation (2), has been chosen to be 0.05 . For this Courant number three runs were performed with different element lengths dx: $0.29 \mathrm{~m}, 0.145 \mathrm{~m}$ and $0.0725 \mathrm{~m}$ in order to see eventual influence of spatial discretisation on calculated pressure surges.

$$
C u=\frac{(a+V) \cdot d t}{d x}
$$

Where $a[\mathrm{~m} / \mathrm{s}]$ is the wave speed, $V[\mathrm{~m} / \mathrm{s}]$ is average velocity of water, $d t[\mathrm{~s}]$ - time step and $d x[\mathrm{~m}]$ element length.

Representative calculation results are shown below in two groups. The first group - Figures 3, 4 and 5 showing calculated pressures at the valve as follows:

- pressures calculated with Relap5Mod3.2.2g and three different element lengths $d x$ in figure 3

- pressures calculated with Relap5Mod3.3patch02 and three different element lengths $\mathrm{dx}$ in figure 4

- pressures calculated with Drako and two different element lengths $\mathrm{dx}(0.2 \mathrm{~m}$ and $0.1 \mathrm{~m})$ in figure 5 .

The second group contains Figures 6, 7 and 8 showing pressures at the distance of $0.25 \mathrm{~L}$ from the high pressure reservoir as follows:

- pressures calculated with Relap5Mod3.2.2g and three different element lengths $d x$ in figure 6

- pressures calculated with Relap5Mod3.3patch02 and three different element lengths $\mathrm{dx}$ in figure 7

- pressures at $0.25 \mathrm{~L}$ calculated with Drako and two different element lengths $\mathrm{dx}(0.2 \mathrm{~m}$ and $0.1 \mathrm{~m})$ in figure 8 .

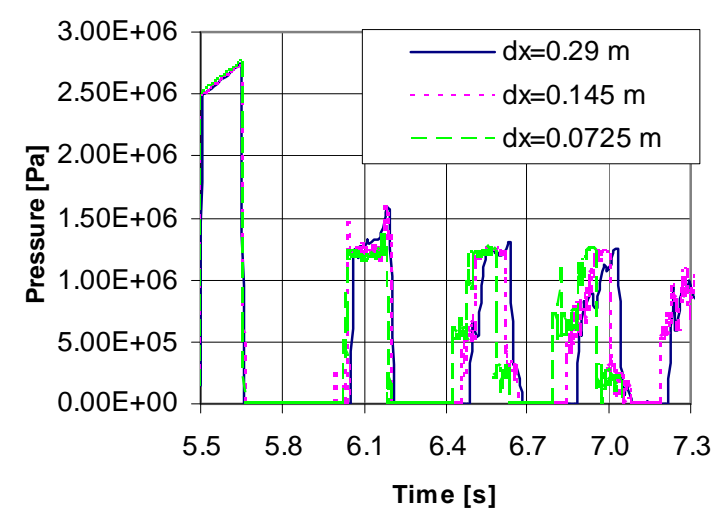

Figure 3. Pressures at the valve (at $x=L$ ) calculated with Relap5Mod3.2.2g with different element lengths $d x$

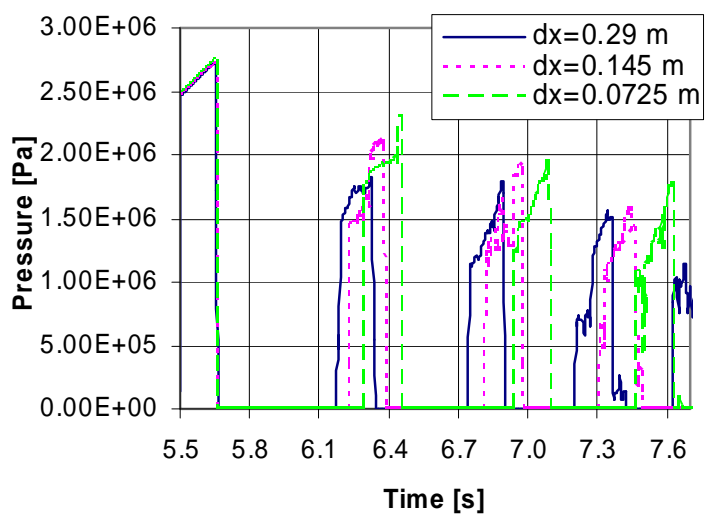

Figure 4. Pressures at the valve (at $x=L$ ) calculated with Relap5Mod3.3patch02 and different element lengths $d x$

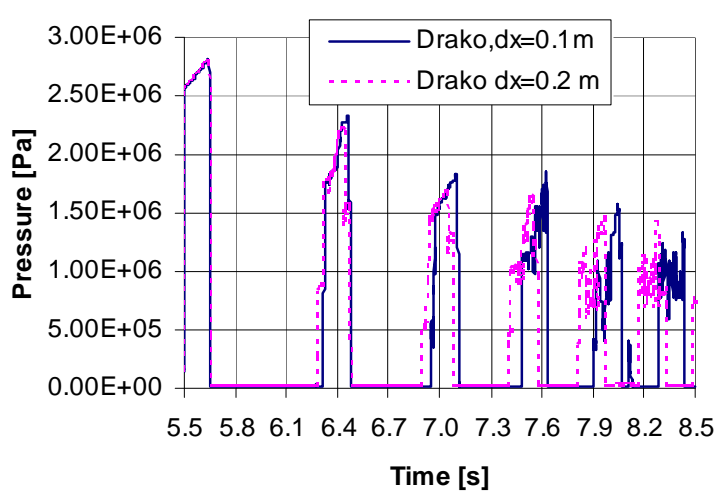

Figure 5. Pressures at the valve (at $x=L$ ) calculated with DRAKO with different element lengths 


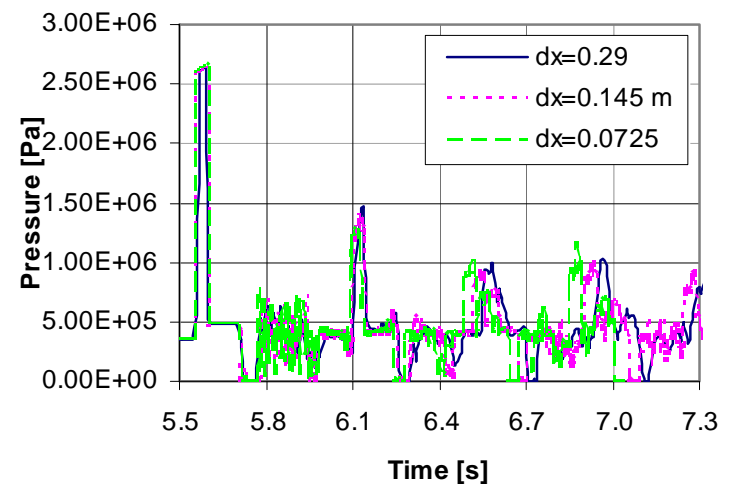

Figure 6. Pressures at $x=0.25 \mathrm{~L}$ calculated with Relap5Mod3.2.2 $g$ with different element lengths $d x$

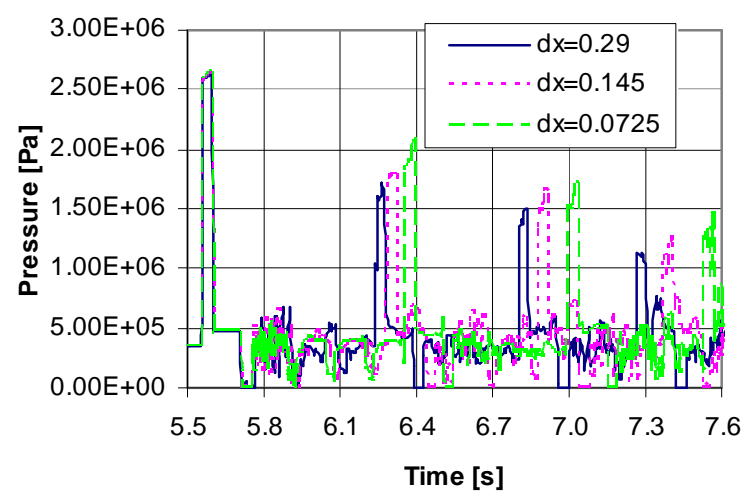

Figure 7. Pressures at $x=0.25 \mathrm{~L}$ calculated with Relap5Mod3.3patch02 with different element lengths $d x$

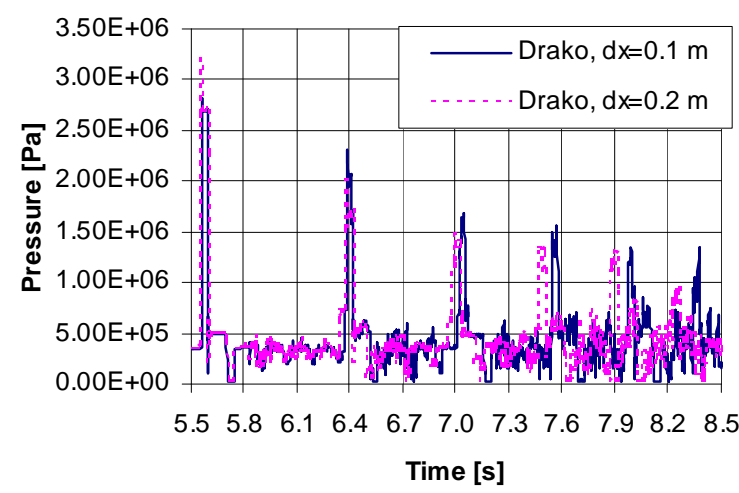

Figure 8. Pressures at $x=0.25 \mathrm{~L}$ calculated with Drako with different element lengths $d x$

Comparison of pressure surges calculated with Relap5Mod3.2.2g (Figure 3 and 6) and Relap5Mod3.3patch02 (Figure 4 and 7) shows following:

- First calculated pressure peaks are identical, amplitudes of second third and fourth peaks calculated by Relap5Mod3.2.2g are much smaller than respective calculated by Relap5Mod3.3p02.

- For both modes of Relap5 the frequency of calculated pressure surges depends on element length. For Relap5Mod3.2.2g frequency is increasing when element length is decreasing, for Relap5Mod3.3patch02 this relation is reversed and stronger.

- Amplitudes of pressure surges calculated with Relap5Mod3.3patch02 are growing when element length $\mathrm{dx}$ becomes shorter.

- Close to the valve the pressure amplitudes given by Drako are similar to results given by Relap5Mod3.3patch02 i.e. amplitudes are slightly influenced by the element length and frequency is decreasing for small element lengths (Figure 5). The relation between Drako and Relap5Mod3.3patch02 is of the same character also at $0.25 \mathrm{~L}$ from the reservoir (Figure 8).

- Pressures calculated by Relap5Mod3.3p02 and Drako are quite similar when comparable element lengths are used.

\subsection{Comparison of calculated and measured pressures}

A preliminary analysis shows that the agreement between the measured pressures and calculated by Relap5Mod3.2.2g is not satisfactory ${ }^{5}$. With exception to first peak are both amplitudes and frequencies in disagreement. Therefore only the calculations performed with Relap5Mod3.3patch02 and Drako will be compared with experimental results and presented below.

Figure 9 shows pressures at the valve (at $x=L$ ) measured and calculated with Relap5 and Drako for Reynolds number $\mathrm{Re}_{0}=30661$ and comparable element length. The differences between the measured and calculated pressure amplitudes are negligible for an engineer. A significant difference regarding time dependency of the calculated pressure surges can be observed. Pressure surges calculated by Drako and Relap5Mod3.3patch02 are almost identical (for comparable element lengths).
${ }^{5}$ Similar conclusion referring to older version of Relap5 was
presented in [9]. 


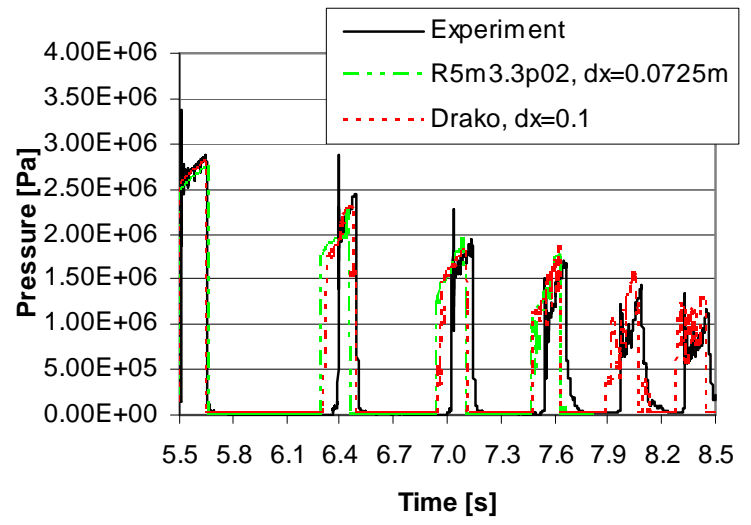

Figure 9. Pressures at the valve measured and calculated with Relap5Mod3.3patch02 and Drako (note that Relap5 calculation has been terminated at $t=7.82 s)$

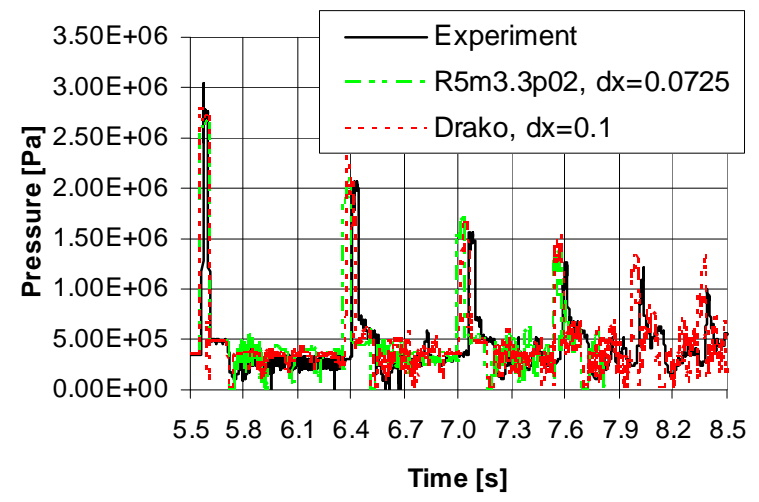

Figure 10. Pressures at distance $0.25 \mathrm{~L}$ from high pressure reservoir measured and calculated with Relap and Drako (note that Relap5 calculation has been terminated at $t=7.82 \mathrm{~s}$ )

\section{CONCLUSIONS}

The calculations of pressure surges in a long pipeline when phase changes occur have been performed with two versions of Relap5 code Relap5Mod3.2.2g and Relap5Mod3.3patch02 and compared with experiments. Calculations were performed for constant Courant number of 0.05 and varying element lengths $\mathrm{dx}$. The pressure surges have also been calculated with computer program Drako and varying element lengths. The results show following:

- Relap5Mod3.2.2g can quite exactly reproduce only the first pressure peak. The pressure peaks coming next diverge from measured values considering both amplitude and time dependency. The frequency of calculated pressure surges is influenced by element length $\mathrm{dx}$ and is increasing when element length is decreasing. The agreement between calculated and measured pressures is not satisfactory. Using Relap5Mod3.2.2g for water hammer calculation with phase changes is therefore not recommended.

- Relap5Mod3.3patch02 can predict not only the first pressure peak but also the following pressure surges. The agreement with measurements is satisfactory regarding pressure amplitudes. Some deviations can be observed regarding time dependency. It should be pointed out that the results are influenced by element length $\mathrm{dx}$. The shortest element length $\mathrm{dx}=0.0725 \mathrm{~m}$ gives the best agreement with experiments. Runs with element length shorter than above mentioned were not performed because of extremely long calculation time. Long calculation time in connection to extremely large amount of produced data is clearly decreasing efficiency of work when Relap5 is used for calculations of steam/water hammer.

- Drako can predict not only the first pressure peak but also the following pressure surges. Also here are the calculated pressure surges dependent on element length $\mathrm{dx}$. The agreement with measurements is satisfactory regarding pressure amplitudes. Some deviations can be observed regarding time dependency. It should be pointed out that the results are probably to some degree influenced by the higher temperature in calculations comparing with experiments.

- Pressure surges calculated by Drako 3.22 and Relap5Mod3.3patch02 are very similar when used element lengths are comparable. For similar element lengths is Drako's calculation time several times shorter than Relap5's.

- Performing the analyses for other cases of waterhammer with water column separation is strongly recommended - such analyses should give basis for estimating an influence of $\mathrm{Re}_{\mathrm{o}}$ and pipe geometry on convergence between calculated and measured pressure courses.

- Numerical parametric studies are also recommended to find element length $\mathrm{dx}$ which is short enough to guarantee the convergence of calculated pressure surges.

\section{REFERENCES}

1. Swedish Nuclear Power Inspectorate (SKI), SKIFS 2005:2, Stockholm, ISSN 1400-1187.

2. Adamkowski A., Lewandowski M., "Experimental Examination of Unsteady Friction Models for Transient Pipe Flow Simulation", Journal of Fluids Engineering, ASME, Vol. 128, No. 6, Nov. 2006, pp.1351-1363.

3. NUREG/CR-5535 "Relap5/MOD3 Code Manual. Volume II: Appendix A Input Requirements", Scientech, Inc. Rockville Maryland Idaho Falls, Idaho, June 1999 (MOD3.2.2Gama). 
4. NUREG/CR-5535 "Relap5/MOD3 Code Manual. Volume V: User's Guidelines”, Scientech, Inc. Rockville Maryland Idaho Falls, Idaho, March 1998 (MOD3.2.2Beta).

5. Tiselj, I., Cerne, G., "Some Comments on the Behaviour of the Relap5 Numerical Scheme at Very Small Tine Steps", Nuclear Science and Engineering: 134, 306-311 (2000).

6. Marcinkiewicz J., Adamkowski A., Lewandowski M., "Experimental Evaluation of Ability of Drako, Flowmaster and Program Using Unsteady Wall Friction Model to Calculate Water Hammer Loadings on Pipelines", ICONE14-89363, Miami, Florida 2006.

7. Kollman D., "DRAKO Pressure Wave Calculation in Pipe Systems With General Components - Short Description", KAE - Kraftverks- und Anlagen Engineering GmbH, 22.11.1999.

8. Wylie, E.B., Streeter, V.L., "Fluid Transients in Systems, Prentince-Hall Inc. New Jersey, 1993.

9. Gale J., Tiselj I., "WAHA (Water Hammer) Computer Code", Proc. of the $9^{\text {th }}$ International Conference on Pressure Surges, BHR Group, Chester, UK, March 2004, pp. 619-631. 